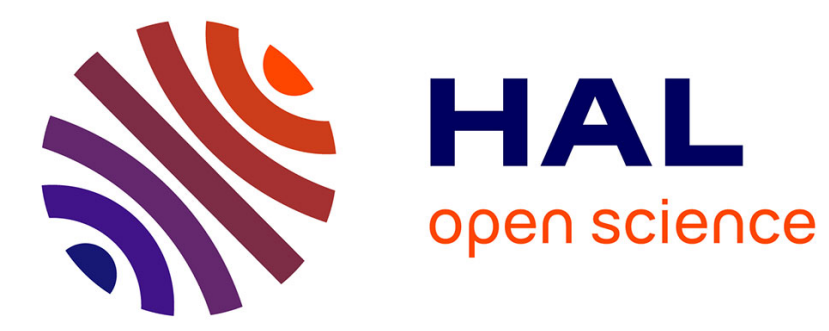

\title{
Barium transport in the hot spot region of fluorescent lamps
}

F Sigeneger, K Rackow, D Uhrlandt, J Ehlbeck, G Lieder

\section{To cite this version:}

F Sigeneger, K Rackow, D Uhrlandt, J Ehlbeck, G Lieder. Barium transport in the hot spot region of fluorescent lamps. Journal of Physics D: Applied Physics, 2010, 43 (38), pp.385201. 10.1088/0022$3727 / 43 / 38 / 385201$. hal-00569711

\section{HAL Id: hal-00569711 \\ https://hal.science/hal-00569711}

Submitted on 25 Feb 2011

HAL is a multi-disciplinary open access archive for the deposit and dissemination of scientific research documents, whether they are published or not. The documents may come from teaching and research institutions in France or abroad, or from public or private research centers.
L'archive ouverte pluridisciplinaire $\mathbf{H A L}$, est destinée au dépôt et à la diffusion de documents scientifiques de niveau recherche, publiés ou non, émanant des établissements d'enseignement et de recherche français ou étrangers, des laboratoires publics ou privés. 


\title{
Barium transport in the hot spot region of fluorescent lamps
}

\author{
F. Sigeneger, K. Rackow, D. Uhrlandt, J. Ehlbeck, G. Lieder \\ INP Greifswald, Felix-Hausdorff-Str. 2, 17489 Greifswald, Germany
}

\begin{abstract}
The transport of barium atoms and ions in the vicinity of the hot spot in fluorescent lamps operating at $25 \mathrm{kHz}$ is investigated by a combined experimental and theoretical approach. By laserinduced fluorescence, the particle densities of barium atoms and ions were measured timeresolved at different distances from the spot center. In addition, the time-dependent cathode fall voltage was measured using an improved band method. The model combines a kinetic part for the electrons with a fluid part for the barium atoms and ions. Both parts are spatially resolved in spherically symmetric geometry. The space-dependent electron Boltzmann equation yields the electron density and the ionization rate coefficient of barium as functions of the cathode fall voltage. These results are used to solve the time-dependent particle balance equations of barium atoms and ions which include the ionization of barium as gain and loss terms, respectively. Good agreement between the measured and calculated particle densities of barium atoms is obtained. A sensitive dependence of the ionization frequency and of the barium particle densities on the cathode fall voltage was found.
\end{abstract}

PACS numbers: 51.50.+v, 52.80.Hc, 52.80.-s, 52.65.-y, 52.70.Kz

Submitted to: Institute of Physics Publishing

J. Phys. D: Appl. Phys., reviced version (19 July 2010) 


\section{Introduction}

Fluorescent lamps play an important role for the energy saving in the light generation. The thermionic electrodes of these lamps are very crucial for an effective lamp operation. These electrodes are double or triple coiled and covered with emitter material which lowers the work function. The emitter material is typically composed of mixtures of alkaline earth oxides with barium as the commonly most important component. As a consequence, currents of several hundred $\mathrm{mA}$ can be delivered by the electrode which works normally in spot mode with temperatures between 1000 and $1500 \mathrm{~K}$ in the spot.

Unfortunately, the emitter material, mainly barium, is transported from the electrode into the plasma volume and finally to the lamp wall or to other surfaces surrounding the electrode. The dominant loss process is the evaporation of barium because of the high temperature at the spot. Sputtering of barium due to the ion bombardment requires cathode fall voltages larger than the threshold energy which is rarely reached during the normal lamp operation.

The loss of emitter material limits the lifetime of fluorescent lamps if these lamps are started with preheated coils and thus the tungsten sputtering as occurring in instant-start lamps is avoided. Therefore, the determination of this loss, especially of barium, is of main interest for the estimation of the lifetime of a lamp. Consequently, a detailed knowledge of the plasma in the cathode region is required.

Several investigations of the cathode region of fluorescent lamps have already been reported in the literature [1-3]. The dynamics of the plasma-electrode coupling in an argon-mercury discharge was experimentally and theoretically investigated in [4]. The model consists of a time-dependent, one-dimensional self-consistent description of the negative glow suplemented by a $0 \mathrm{~d}$ sheath model at the cathode side and a $0 \mathrm{~d}$ model for the positive column. The transition from the localized spot to the negative glow covering the full tube is approximately taken into account by the corresponding ratio of surface and cross sectional areas.

A two-dimensional model of a helium-xenon dc discharge have been presented in [5]. The model spacially resolves the sheath in front of the spot and describes the transition of the discharge from the spot through the negative glow towards the anode.

The current paper describes an somewhat alternative approach which is more concentrated on the barium transport. For this purpose, experimental and theoretical investigations at a fluorescent model lamp driven at $25 \mathrm{kHz}$ have been performed. The densities of Ba atoms and ions at different distances from the spot center have been measured time-resolved by laser induced fluorescence (LIF). Furthermore, the time-dependent cathode fall voltage was approximately determined using an improved band method. A model is applied to deduce the net flux of barium from the cathode surface. The model comprises the solution of the time-dependent particle balance equations of $\mathrm{Ba}$ and $\mathrm{Ba}^{+}$which include the Ba ionization as gain and loss terms, respectively. The ionization rate coefficient of $\mathrm{Ba}$ and the electron density are determined by solving the space-dependent electron Boltzmann equation using the measured cathode fall voltage and the discharge current as input. As a result, the time-dependent particle flux of barium atoms and ions is obtained by the model.

The paper is organized as follows. The experimantal investigations are reported in section 2. In section 3 the model including the kinetic and the fluid part are described and the results are presented in section 4 . 


\section{Experiments}

\subsection{Model lamp}

A fluorescent lamp identical to commercial T8 - $36 \mathrm{~W}$ lamps is used for the investigation. The lamp is $120 \mathrm{~cm}$ in length and $25 \mathrm{~mm}$ in diameter. It contains mercury and a mixture of $90 \%$ argon and $10 \%$ krypton at a pressure p of $210 \mathrm{~Pa}$. As electrode a so-called stick coil is used which is designed for an rms lamp current of $320 \mathrm{~mA}$. The electrode is carried by two posts which are positioned in a distance of about $10 \mathrm{~mm}$. To enable transmission of the laser beam and the fluorescent light for the LIF, the glass tubes have been cleared of phosphor. A sketch of the electrode region of the fluorescent lamp and a typical visual observation of the spot is shown in Fig. 1.

(a)

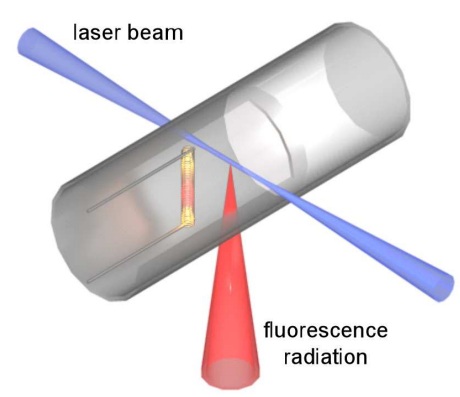

(b)

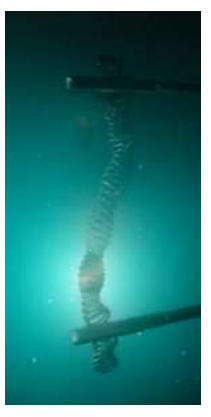

Figure 1. Sketch of the cathode region in the fluorescent lamp (a) and typical observation of the spot at the lower end of the coil (b).

To generate a lamp current comparable to commercial ballasts, a current controlled power supply working at $25 \mathrm{kHz}$ is used. The lamp voltage and current are measured by a high voltage differential probe and a high frequency current probe, respectively.

\subsection{LIF-measurements}

The density of barium atoms and ions in ground state is measured by laser induced fluorescence in a short distance from the spot. This distance is varied in steps of $0.5 \mathrm{~mm}$ in axial direction of the tube. The laser radiation at $350.1 \mathrm{~nm}$ excites the barium atom from the ground state $6 \mathrm{~s}^{2}{ }^{1} \mathrm{~S}_{0}$ to the upper level $5 \mathrm{~d} 6 \mathrm{p}^{1} \mathrm{P}_{1}$. This level decays spontaneously via different channels as indicated in Fig. 2. The detected fluorescent radiation at $582.6 \mathrm{~nm}$ from the transition to the $6 \mathrm{~s} 5 \mathrm{~d}^{1} \mathrm{D}_{2}$ level is used to determine the population density of the ground state. In case of barium ions the excitation from ground state $6 \mathrm{~s}^{2} \mathrm{~S}_{1 / 2}$ to $6 \mathrm{p}^{2} \mathrm{P}_{3 / 2}(455.4 \mathrm{~nm})$ and the spontaneous transition to the $5 \mathrm{~d}^{2} \mathrm{D}_{5 / 2}$ level is used. The atomic data were taken from [6].

The setup of the LIF measurement is shown in Fig. 3. For the generation of the laser beam, a Nd:YAG pumped dye laser operating at $10 \mathrm{~Hz}$ is used. In case of barium atoms, the dye laser is pumped at $532 \mathrm{~nm}$. It provides radiation at $700.2 \mathrm{~nm}$ which is frequency doubled by a KDP crystal to the desired $350.1 \mathrm{~nm}$. In case of barium ions, the dye laser is pumped at $355 \mathrm{~nm}$ and provides the desired $455.4 \mathrm{~nm}$ directly. The output beam is expanded by a Galilean telescope. For defining the beam diameter, the beam is directed through a $1 \mathrm{~mm}$ aperture which covers only a small region of the original beam. A spatial filter is used to restore the resulting degraded beam profile to a nearly Gaussian shape. It consist of two lenses ( $\mathrm{f}=100 \mathrm{~mm}$ each) in a distance of $200 \mathrm{~mm}$ with a $20 \mu \mathrm{m}$ pinhole located at the common focal point. The resulting beam is directed perpendicular to 


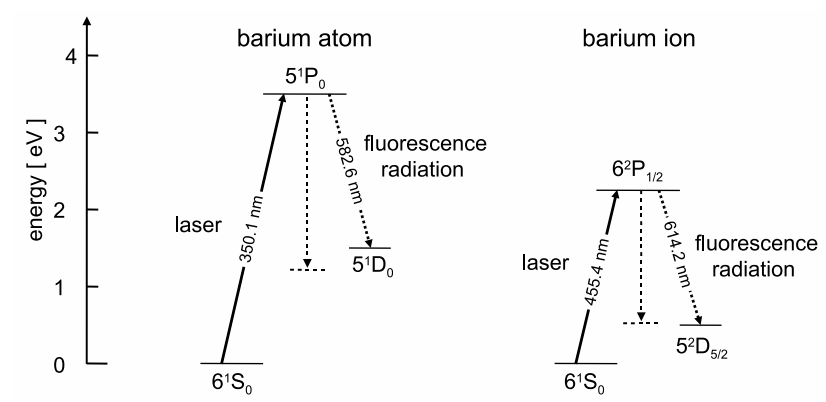

Figure 2. Radiation transitions used for the LIF measurements of barium atom and ions. The dotted lines symbolize radiative transitions to other levels which are not used for the measurements.

the glass surface through the lamp directly in front of the coil as illustrated in Fig. 1. Its intensity is measured by a photodiode.

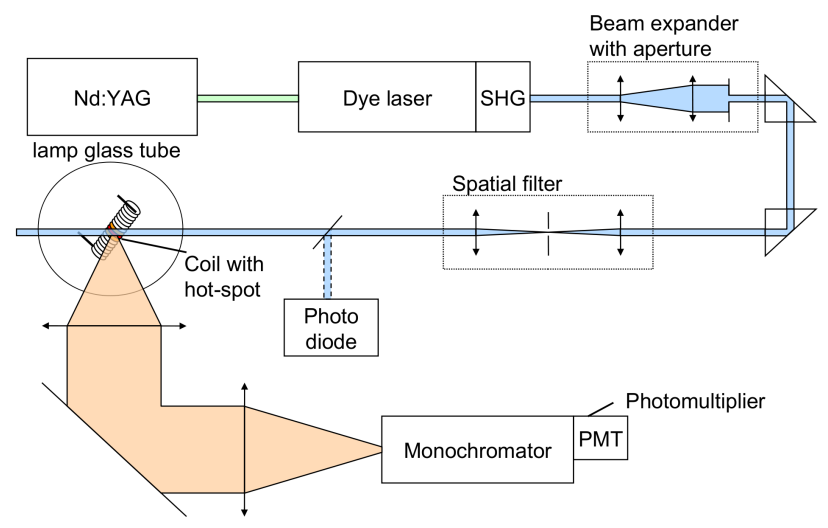

Figure 3. Setup for the LIF measurement.

The lamp is movable in three dimensions by stepping motors which allows to adjust the distance from the hot spot to the beam. The position of the coil is determined by moving it through the laser beam and observing the shadowing. As the beam has a diameter of $1 \mathrm{~mm}$, the minimal distance from coil to beam is approximately $0.5 \mathrm{~mm}$. The fluorescent radiation at $582.6 \mathrm{~nm}$ is observed perpendicular to the beam. The region of interest is imaged (1:3) to the entrance slit of a $0.5 \mathrm{~m}$ monochromator (Acton Research SpectraPRO-500I) with a 1800 grooves-per-millimeter grating. To attenuate the scattered laser light, a long pass filter is placed in front of the entrance slit. The fluorescent radiation is measured using a photomultiplier and averaged over an observation volume of about $0.7 \times 0.7 \times 1.0 \mathrm{~mm}^{3}$. In general, the fluorescent signal is averaged over 600 laser pulses. The Rayleigh calibration technique was applied to determine absolute barium atom densities. This commonly used method is described in text books. Hadrath [7] applied this method to measure absolute tungsten atom densities. Because of the strong dependence of the Rayleigh scattering intensity on the wavelength, this calibration method could not successfully be applied for the barium ion measurements.

\subsection{Cathode fall measurements}

For determination of the cathode fall voltage, the so-called band technique is used $[8,9]$. As shown schematically in Fig. 4, a copper band is wounded around the outside of the glass tube in the electrode region and the voltage between band and electrode is measured. This voltage represents 


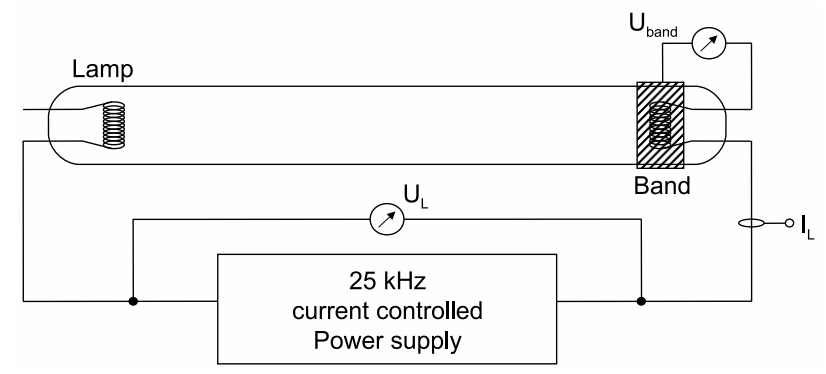

Figure 4. Electrical setup for lamp operation and cathode fall measurement.

the capacitively coupled wall potential and includes the cathode fall, the voltage drop in the plasma from axis to wall and the wall sheath. To deduce the cathode fall voltage from this measurement some considerations are required. Garner has investigated [8] the relation between the various voltage drops inside the discharge and the voltage measured at the band for conditions which are similar to those considered here. It was shown that the band voltage can be used as a measure for the cathode fall voltage if an appropriate correction factor is taken into account. The measuring circuit consists of the band capacity with respect to the inner wall and a resistor and capacity inside the probe. This circuit represents a high pass filter with a characteristic frequency response which has to be considered for the used frequency. At $25 \mathrm{kHz}$ a correction factor of 1.2 is applied to the measured voltage in correspondence to comparisons with internal probe measurements [8]. The high pass characteristic also eliminates the DC part of the potential. Therefore, we assume a cathode fall equal to zero at the beginning of the cathode phase (lamp voltage is equal to zero) by adding a DC voltage. Figure 5 shows the measured band voltage for two different lamp currents as well as the used scaled and shifted voltage.
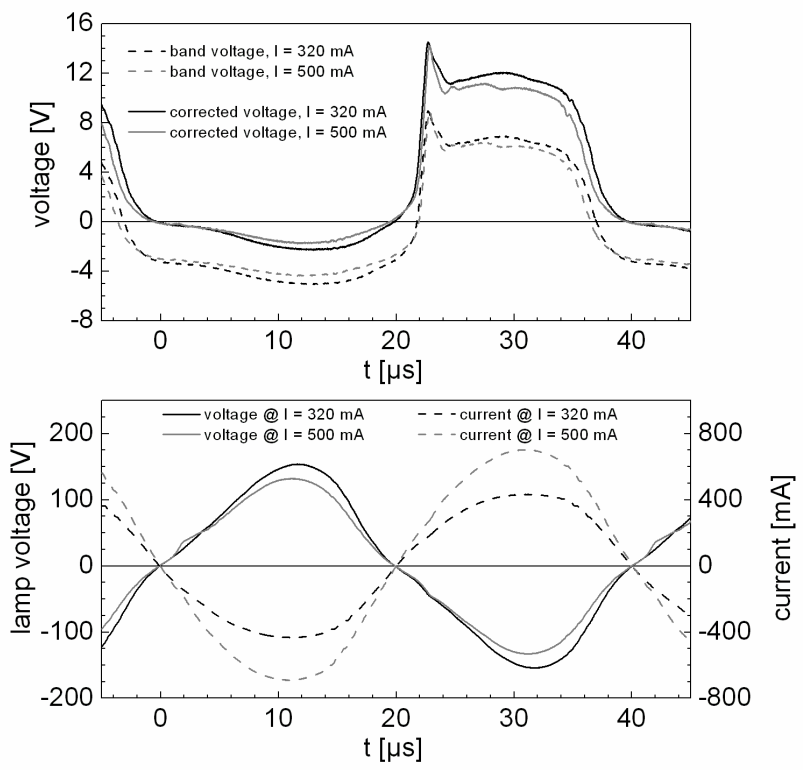

Figure 5. Measured band voltage and corrected voltage used as cathode fall. (top) Lamp voltage and lamp current. (bottom) 


\section{Barium transport model}

\subsection{Electron kinetics}

The main impact of the plasma on the barium transport is the ionization of barium atoms. Therefore, the ionization frequency $\nu_{B a}^{i o}$ must be determined in dependence on the cathode fall voltage $U_{\mathrm{CF}}$. For this purpose, the space-dependent electron Boltzmann equation

$\vec{v} \cdot \nabla_{\vec{r}} f-\frac{e_{0}}{m} \mathbf{E} \cdot \nabla_{\vec{v}} f=\mathcal{C}^{\mathrm{el}}+\sum_{k, l} \mathcal{C}_{k, l}^{\mathrm{ex}}+\sum_{k} \mathcal{C}_{k}^{\text {io }}$

is solved which includes the electric field $\mathbf{E}$ and the action of elastic, exciting and ionizing collisions of the electrons with the gas atoms in the related collision integrals $\mathcal{C}^{\mathrm{el}}, \mathcal{C}_{k, l}^{\mathrm{ex}}$ and $\mathcal{C}_{k}^{\text {io }}$. Here, $e_{0}$ and $m$ denote the electron charge and mass. The threshold energies of the inelastic processes which have been included are listed in Table 1. The excitation cross section for argon were taken from Ref. [10] and the ionization cross section for argon as well as all cross sections for krypton from Ref. [11]. The cross section set for mercury is the same as that used in Ref. [12]. For barium, the ionization cross section was taken from Ref. [13].

\begin{tabular}{cl|rl|rl|rl}
\hline \multicolumn{2}{c|}{$\mathrm{Ar}$} & \multicolumn{2}{|c|}{$\mathrm{Kr}$} & \multicolumn{2}{c}{$\mathrm{Hg}$} & \multicolumn{2}{c}{$\mathrm{Ba}$} \\
$U^{\text {in }}[\mathrm{eV}]$ & level & $U^{\text {in }}[\mathrm{eV}]$ & level & $U^{\text {in }}[\mathrm{eV}]$ & level & $U^{\text {in }}[\mathrm{eV}]$ & level \\
\hline 11.55 & $1 \mathrm{~s}_{5}$ & 9.91 & $1 \mathrm{~s}_{4+5}$ & 4.66 & $6^{3} \mathrm{P}_{0}$ & 5.24 & ion \\
11.62 & $1 \mathrm{~s}_{4}$ & 10.56 & $1 \mathrm{~s}_{2+3}$ & 4.87 & $6^{3} \mathrm{P}_{1}$ & & \\
11.72 & $1 \mathrm{~s}_{3}$ & 11.30 & $2 \mathrm{p}_{5-10}$ & 5.43 & $6^{3} \mathrm{P}_{2}$ & & \\
11.82 & $1 \mathrm{~s}_{2}$ & 12.00 & $\mathrm{Kr}^{*}$ & 6.68 & $6^{1} \mathrm{P}_{1}$ & & \\
12.90 & Ar* & 14.00 & ion & 11.00 & $\mathrm{Hg}^{*}$ & & \\
15.76 & ion & & & 10.44 & ion & & \\
\hline
\end{tabular}

Table 1. Collision cross sections used for solving the electron Boltzmann equation. Ar*, $\mathrm{Kr}^{*}$ and $\mathrm{Hg}^{*}$ denote lumped levels of higher excited states.

Visual observations of the spot as shown in Fig. 1b suggest that the plasma is largely spherically symmetric within the near vicinity of the spot. Therefore, a one-dimensional spherically symmetric approach [14] is chosen to solve eqn. (1). Taking into account the radially directed electric field $\mathbf{E}=E_{r} \mathbf{e}_{r}$, the electron velocity distribution function $\bar{f}(\mathbf{v}, r)$ can be expanded with respect to the ratio $v_{r} / v \equiv v_{r} /|\mathbf{v}|$ in Legendre polynomials. In the lowest approximation order, the two-term approximation, the velocity distribution is represented by

$f\left(u, \frac{v_{r}}{v}, r\right)=\frac{1}{2 \pi}\left(\frac{m}{2}\right)^{\frac{3}{2}}\left[f_{0}(u, r)+f_{r}(u, r) \frac{v_{r}}{v}\right]$

where $f_{0}(u, r)$ and $f_{r}(u, r)$ are the isotropic and anisotropic parts of the velocity distribution function and $u=m v^{2} / 2$ denotes the electron kinetic energy. As detailed in [14], the electron Boltzmann equation is transformed using (2) into a partial differential equation with shifted energy arguments which is solved as an initial boundary value problem from higher to lower energies. The spatial solution region spans from the approximated radius of the spot $r_{c}$ up to the radius $r_{a}$ of an assumed outer virtual spherical anode.

Because the cathode fall region in front of a thermionic cathode is very thin the spatial profile of the electric field within this region is not resolved in the kinetic treatment. Instead, the action of the cathode fall voltage $U_{\mathrm{CF}}$ is taken into account in the boundary condition. The Gaussian-like function

$\hat{f}_{r}(u)=c u \exp \left[-\left(\frac{u-e_{0} U_{\mathrm{CF}}}{\Delta u}\right)^{2}\right]$ 
is prescribed to the anisotropic distribution part $f_{r}\left(u, r_{c}\right)$ at the cathode position $r_{c}$. This function models the influx of electrons into the plasma which have been released from the cathode due to thermionic emission and accelerated in the cathode fall up to the energy $e_{0} U_{\mathrm{CF}}$ with an energetic half-width of $\Delta u$. The factor $c$ is used to normalize the solution $f(u, r)$ in the whole solution region. It is determined as detailed below. In the plasma volume a very low electric field strength $E_{0}$ is assumed.

From the solution of the Boltzmann equation the electron density $n_{e}$ and the ionization rate coefficient $k_{\mathrm{Ba}}^{\mathrm{io}}$ are calculated according to

$n_{e}^{(0)}(r)=\int_{0}^{\infty} u^{1 / 2} f(u, r) d u$

$k_{\mathrm{Ba}}^{\mathrm{io}}(r)=\frac{1}{n_{e}^{(0)}(r)} \sqrt{\frac{2}{m_{e}}} \int_{0}^{\infty} u Q_{\mathrm{Ba}}^{\mathrm{io}}(u) f_{0}(u) d u$

Similarly, the rate coefficients $k_{\mathrm{A} r}^{\mathrm{io}}, k_{\mathrm{K} r}^{\mathrm{io}}$ and $k_{\mathrm{H} g}^{\mathrm{io}}$ for the ionization of argon, krypton and mercury atoms are calculated. To get the normalization factor $c$, additionally the current through the shell $i_{e}(r)=4 \pi e_{0} r^{2} \phi_{r}(r)$ is calculated where the particle flux $\phi_{r}$ depends on the anisotropic distribution part according to

$\phi_{r}(r)=\frac{1}{3} \sqrt{\frac{2}{m}} \int_{0}^{\infty} f_{r}(u, r) u d u$.

The electron particle balance equation reads

$$
\begin{gathered}
\frac{1}{r^{2}} \frac{d}{d r}\left(r^{2} \phi(r)\right)=S_{e}(r)=n_{e}^{(0)}(r) \sum_{\alpha} N_{\alpha}(r) k_{\alpha}^{\mathrm{io}}(r), \\
\alpha=\{A r, K r, H g, B a\}
\end{gathered}
$$

and yields in integrated form the relation

$i_{e}(r)=i_{e}\left(r_{c}\right)+4 \pi e_{0} \int_{r_{c}}^{r} S_{e}(\bar{r}) \bar{r}^{2} d \bar{r}$

At larger distances from the spot the source $S_{e}$ becomes very small and the current $i_{e}$ through the shell is nearly independent of $r$. At these positions, the contribution of the ion current can be neglected. Therefore, the relation $i_{e}\left(r_{a}\right)=i_{L}^{(0)}$ is used to determine the factor $c$ in (3) and to normalize the results on a fixed discharge current $i_{L}^{(0)}$.

The collision integrals in (1) include the particle densities of the various gas atoms in the discharge. Due to the large temperature of the cathode in the spot also the increased gas temperature and the resultant decrease of the gas density must be taken into account. This effect is approximately included by solving the simplified heat balance equation $\Delta T=0$ in spherical symmetry which yields the result $T=\frac{c_{1}}{r}+c_{2}$. The temperature $T_{S}$ at the spot and $T_{\infty}$ far away from the spot are used to determine the coefficients $c_{1}$ and $c_{2}$.

Some results derived from the solution of the electron Boltzmann equation are shown in Figs. 6-7. These results have been obtained for a discharge in a mixture of $\alpha_{\mathrm{Ar}}=90 \%$ argon and $\alpha_{\mathrm{Kr}}=10 \%$ krypton at a pressure $p$ of $210 \mathrm{~Pa}$. The $\mathrm{Hg}$ vapor pressure is determined according to $\ln p_{\mathrm{H} G}\left(T_{\mathrm{H} G}\right)=30.804-0.8254 \ln T_{\mathrm{H} G}-7564 / T_{\mathrm{H} G}$ with $p_{\mathrm{H} G}$ in mTorr [15]. A cold spot temperature of $T_{\mathrm{H} G}=300 \mathrm{~K}$ was used. The spot radius $r_{c}$ and the field in the negative glow $E_{0}$ are assumed to be $0.5 \mathrm{~mm}$ and $0.2 \mathrm{~V} / \mathrm{cm}$, respectively. A radius $r_{a}=2 \mathrm{~cm}$ was taken for the spherical virtual anode. The energetic half-width of the influx profile (3) was assumed to be $0.2 \mathrm{eV}$.

In Fig. 6 the isotropic part of the electron velocity distribution function is shown as function of the kinetic energy $u$ and the radius $r$ for a cathode fall voltage $U_{\mathrm{CF}}$ of 10 and $20 \mathrm{~V}$. The group of 
primary electrons has a mean energy which is related to the cathode fall voltage. Further groups of electrons are generated due to inelastic collisions with the gas atoms. The energy gaps between the groups correspond to the various threshold energies listed in Table 1. As shown, a very complex distribution function is generated due to the superimposed action of inelastic collisions. Similar electron distribution functions, but with somewhat less pronounced structures have been observed by Monte Carlo simulations [16] and by probe measurements [17] in the cathode region of glow discharges. Furthermore, the ionization cross section of barium $Q_{\mathrm{B} a}^{\mathrm{io}}$ is included in Fig. 6. The integral over both quantities shown in Fig. 6 according to (5) yields the ionization rate coefficient $k_{\mathrm{Ba}}^{\mathrm{io}}$ as function of the radius $r$ and the cathode fall voltage $U_{\mathrm{CF}}$.

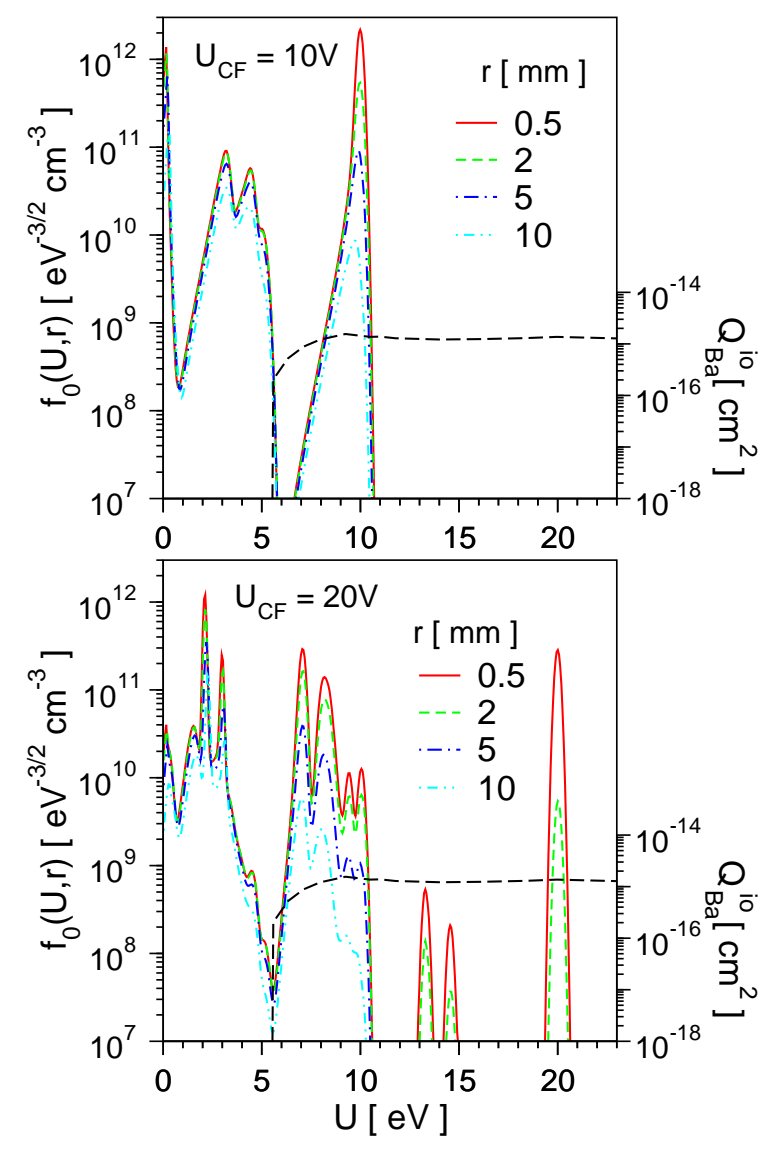

Figure 6. Isotropic electron velocity distribution part $f_{0}$ and ionization cross section of $\mathrm{Ba} Q_{\mathrm{B} a}^{\mathrm{i} o}$.

The result is shown in Fig. 7 together with the electron density. Due to the complex structure of the distribution function a non-monotonous dependence of both quantities on $U_{\mathrm{CF}}$ was obtained. Especially, a pronounced minimum around $U_{\mathrm{CF}}=15 \mathrm{~V}$ can be observed in the ionization rate coefficient which is important for the modelling of the barium transport.

\subsection{Ba transport model}

The transport of the barium atoms and ions is investigated by solving the particle balance equations $\frac{\partial}{\partial t} n_{a}+\nabla \cdot \Gamma_{a}=-\nu_{\mathrm{Ba}}^{\text {io }}(r) n_{a}$

$\frac{\partial}{\partial t} n_{i}+\nabla \cdot \boldsymbol{\Gamma}_{i}=\nu_{\mathrm{Ba}}^{\text {io }}(r) n_{a}$

which include the time- and space-dependent densities $n_{a}$ and $n_{i}$ of barium atoms and ions. The atom flux $\boldsymbol{\Gamma}_{a}=-D^{\mathrm{Ba}} \nabla n_{a}$ and the ion flux $\boldsymbol{\Gamma}_{i}=\mu_{i} n_{i} \mathbf{E}-D^{\mathrm{Ba}} \nabla n_{i}$ are determined by the diffusion 

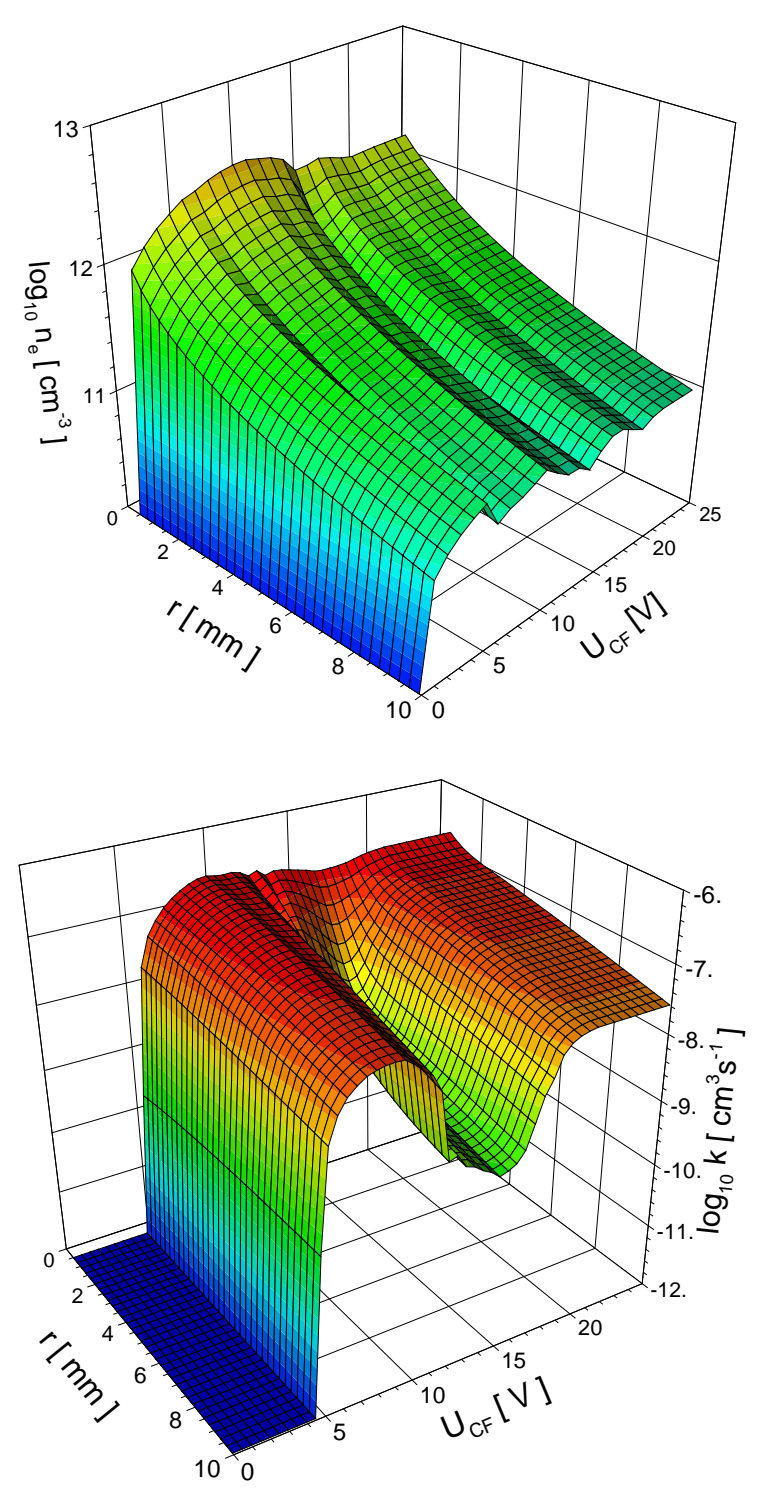

Figure 7. Electron density and Ba ionization rate coefficient as functions of $U_{\mathrm{CF}}$. The electron density was normalized on a discharge current of $500 \mathrm{~mA}$.

coefficient $D^{\mathrm{Ba}}$, the mobility $\mu_{i}$ and the electric field strength E. Similar to the kinetic equation, the balance equations (9)-(10) are solved in one dimension assuming spherical symmetry.

The standard diffusion coefficients $D_{0}^{\mathrm{Ba}, \mathrm{Ar}}$ and $D_{0}^{\mathrm{Ba}, \mathrm{Kr}}$ of barium ground state atoms in argon and krypton were experimentally determined in [18]. The diffusion coefficient $D_{0}^{\mathrm{Ba}}$ of barium atoms in the gas mixture of argon and krypton was calculated using Blank's law

$D_{0}^{\mathrm{Ba}}=\left(\frac{\alpha_{\mathrm{Ar}}}{D_{0}^{\mathrm{Ba}, \mathrm{Ar}}}+\frac{\alpha_{\mathrm{Kr}}}{D_{0}^{\mathrm{Ba}, \mathrm{Kr}}}\right)^{-1}$

which results in $D_{0}^{\mathrm{Ba}}=0.113 \mathrm{~cm}^{2} / \mathrm{s}$. This standard diffusion coefficient is related to the standard temperature $T_{0}=273 \mathrm{~K}$ and pressure $p_{0}=760$ Torr or the related density $N_{0}=2.687 \cdot 10^{19} \mathrm{~cm}^{-3}$. The actual diffusion coefficient $D^{\mathrm{Ba}}(r)$ for barium near the spot is determined by the relation [18]

$$
D^{\mathrm{Ba}}(r)=D_{0}^{\mathrm{Ba}}\left(\frac{p_{0}}{p}\right)\left(\frac{T(r)}{T_{0}}\right)^{3 / 2}
$$

where the dependence of the gas temperature $T$ on the distance $r$ from the spot center is taken into account. For the mobility $\mu_{i}$ of barium ions a field dependent standard mobility 
$\mu_{i, 0}(|\mathbf{E}|)$ has been taken from [19]. The space-dependent temperature is considered by the relation $\mu_{i}(r)=\mu_{0}(|\vec{E}|) N_{0} / N(r)$ with $N(r)=p /\left(k_{\mathrm{B}} T(r)\right)$.

The impact of the plasma on the barium transport is mainly caused by the ionization of barium atoms with the ionization frequency $\nu_{\mathrm{Ba}}^{\mathrm{io}}$. This time- and space-dependent quantity is deduced in the cathode phase according to

$\nu_{\mathrm{Ba}}^{\text {io }}(t, r)=k_{\mathrm{Ba}}^{\mathrm{io}}\left(U_{\mathrm{CF}}(t), r\right) n_{e}(t, r)$

by using the measured temporal evolution of the cathode fall voltage $U_{\mathrm{CF}}(t)$. The time- and spacedependent electron density is determined by the relation $n_{e}(t, r)=n_{e}^{(0)}\left(U_{\mathrm{CF}}(t), r\right) i_{L}(t) / i_{L}^{(0)}$ using the measured discharge current $i_{L}(t)$ and the electron density $n_{e}^{(0)}\left(U_{\mathrm{CF}}, r\right)$ which is calculated in the electron kinetic treatment and normalized on the fixed discharge current $i_{L}^{(0)}$. In the anode phase the barium ionization frequency is assumed to be negligible. This approach corresponds to the quasi-stationary solution of the electron Boltzmann equation and neglects the delayed response of the electron density as observed e.g. by Garner [4]. Nevertheless, the ionization frequency $\nu_{\mathrm{Ba}}^{\text {io }}$ is rapidly droped down during the transition from the cathode to the anode phase due to the diminishing cathode fall voltage.

Appropriate boundary conditions have to be chosen at the spot $\left(r=r_{c}\right)$ and the virtual anode $\left(r=r_{a}\right)$. At the spot a constant flux $\Gamma_{\mathrm{a}}^{\mathrm{c}}$ is prescribed to the flux of barium atoms. This corresponds to the assumption of a time independent temperature profile of the electrode surface in the spot. The determination of the barium atom flux generally requires a detailed description of the Ba diffusion in the emitter and the evaporation process. To avoid possible errors due to the insufficience of existing models and limited knowledge of corresponding data, the value of $\Gamma_{\mathrm{a}}^{\mathrm{c}}$ is used as a scaling factor. Including the measured densities of $\mathrm{Ba}$ atoms in absolute units, the whole solution is scaled to reproduce on average the measured atom densities. This procedure allows finally to determine the value of the evaporation flux independent of the treatment of the evaporation process itself.

For the barium ions the boundary condition

$$
\boldsymbol{\Gamma}_{i} \cdot \mathbf{n}=\frac{1-R}{1+R}\left(\mu_{i} \mathbf{E}_{\mathrm{S}} \cdot \mathbf{n} n_{i}+v_{i}^{\mathrm{th}} n_{i} / 2\right)
$$

was used [20] where $\mathbf{n}$ denotes the outward directed normal vector, $R \approx 0.1$ the reflection coefficient and $v_{i}^{\text {th }}=\sqrt{\frac{8 k_{\mathrm{B}} T}{\pi m_{i}}}$ the ion thermal velocity. The surface electric field $E_{\mathrm{S}}$ at the cathode surface was approximated using a sheath model [4] according to

$$
E_{\mathrm{S}}^{2}(t)=2\left(\frac{k_{\mathrm{B}} T_{e}^{0}}{e_{0} \lambda_{D}(t)}\right)^{2}\left(\sqrt{1+2 \eta_{\mathrm{sh}}(t)}+e^{-\eta_{\mathrm{sh}}(t)}-2\right)
$$

with the Debye length $\lambda_{D}(t)=\sqrt{\varepsilon_{0} k_{\mathrm{B}} T_{e}^{0} /\left(n_{e}(t, 0) e_{0}^{2}\right)}$ and $\eta_{\mathrm{sh}}(t)=e_{0} U_{\mathrm{CF}}(t) /\left(k_{\mathrm{B}} T_{e}^{0}\right)$. The electron temperature $T_{e}^{0}$ at the plasma/sheath boundary was approximated by a value of one $\mathrm{eV}$.

To determine the drift flux of the barium ions the electric field $\mathbf{E}$ in the plasma volume is required. This field could be determined by a self-consistent model including all ions and the Poisson equation. However, such a model is not the subject of the current investigation. Therefore, the constant value $\mathbf{E}=E_{0} \mathbf{e}_{r}$ in accordance with the kinetic part is used. The influence of this parameter on the ion density and flux is discussed below.

The radius $r_{a}$ of the virtual anode was chosen much larger than the region around the spot where remarkable barium densities can be detected. Therefore, simple boundary conditions $n_{a}\left(r_{a}\right)=0$ and $n_{i}\left(r_{a}\right)=0$ are used there because the desired results near the spot are not sensitive to these conditions. 


\section{Results}

Using the corresponding time-dependent electrode fall voltage from the measurements, electron kinetic and transport equations are solved to determine the Ba atom and ion densities. The solution is scaled to meet the atom densities measured by calibrated LIF on average. The time-dependent calculated courses of the $\mathrm{Ba}$ atom densities at different distances from the spot are compared in Fig. 8 with corresponding results which have been obtained from the LIF measurements for a discharge current of $i_{L}=320$ and $500 \mathrm{~mA}$, respectively. The measured LIF results represent densities averaged over the finite cuboid observation volume of about $0.7 \times 0.7 \times 1.0 \mathrm{~mm}^{3}$. The deviation of the local atom density from the spatially averaged one was checked using the calculated radial density profile for $i_{L}=500 \mathrm{~mA}$ as shown in Fig. 8 . If using the period averaged profile, the local density at $r=1 \mathrm{~mm}$ is about $2 \%$ larger than the spatially averaged one and about $14 \%$ lower if using the steepest profile in the cathode phase $(t=30 \mu \mathrm{s})$. Good agreement of the calculated time-dependent atom densities with the measured results was obtained for both discharge currents at different distances from the spot center. The figures illustrate the decrease of the atom densities in the cathode phase caused by the Ba ionization. During the anode phase the atom density again increases due to the diffusion of atoms from the cathode. The decrease of the atom density is more pronounced at $500 \mathrm{~mA}$ than at $320 \mathrm{~mA}$ because of the larger electron density and corresponding increased ionization rate.
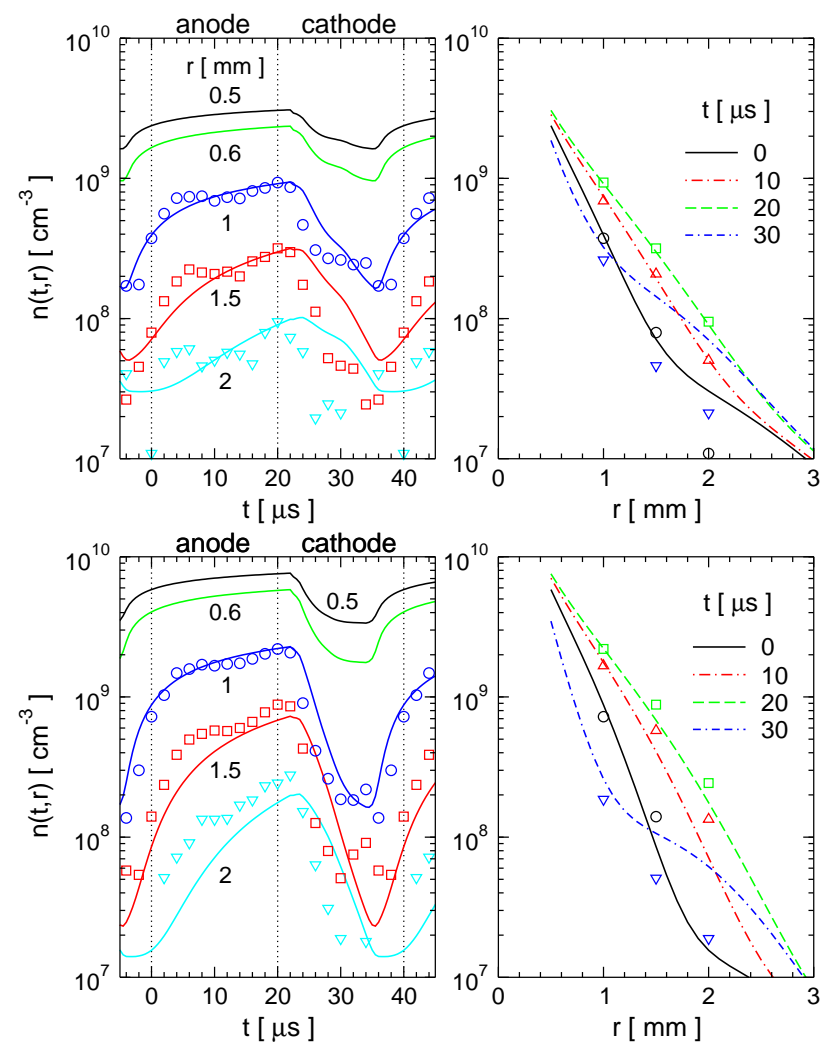

Figure 8. Ba atom densities for $i_{L}=320$ (top) and $500 \mathrm{~mA}$ (bottom) as function of time and the radial coordinate. The calculated results (curves) are compared with the measured ones (symbols).

The ion density is shown in Fig. 9 for both discharge currents. As to be seen, the decrease of the atom density in the cathode phase coincides with an increase of the ion density which is available in arbitrary units only. As shown in Fig. 9, the measured modulation of the ion density agrees well with the model result. The radial profiles as shown in the right diagrams of Fig. 9 have 
a local maximum due to the absorption of ions at the cathode. The ion density at the cathode is strongly decreased in the cathode phase because of the increased electric field.
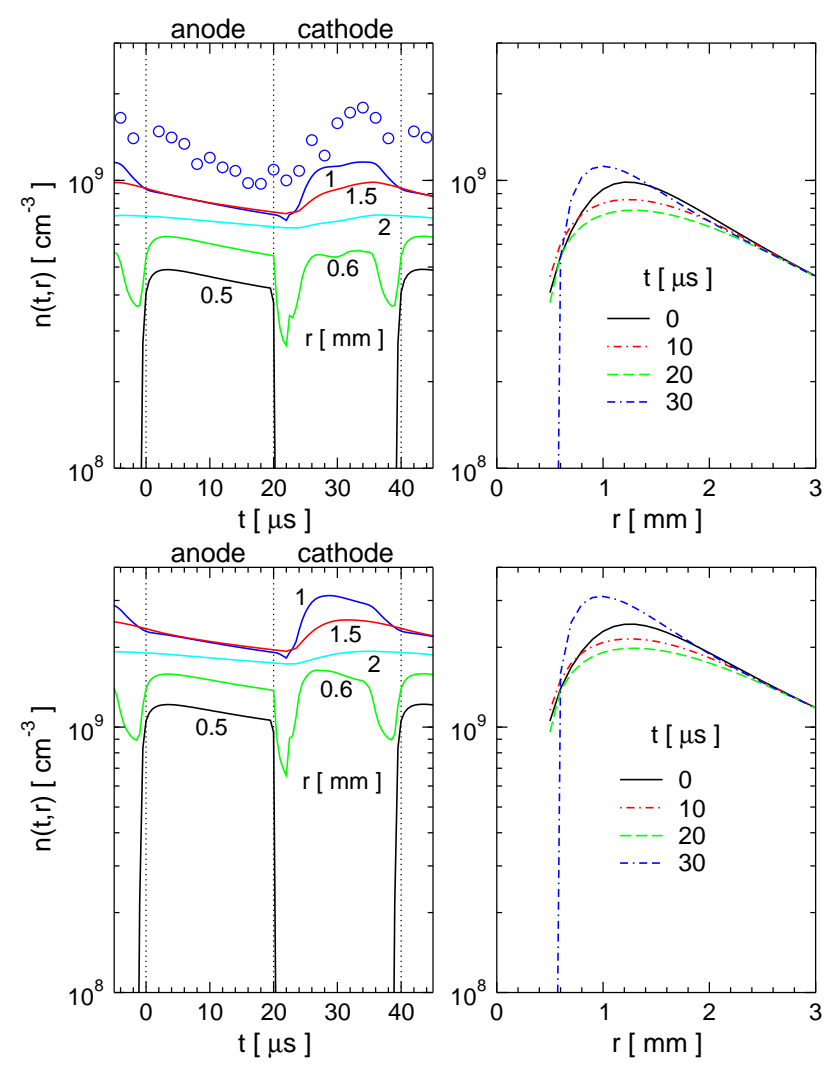

Figure 9. Ba ion densities for $i_{L}=320$ (top) and $500 \mathrm{~mA}$ (bottom). The measured ion density (circles) for $320 \mathrm{~mA}$ and distance $1 \mathrm{~mm}$ is shown in arbitrary units.

The particle fluxes of atoms and ions are shown in Fig. 10. The time-independent atom fluxes $\Gamma_{\mathrm{a}}^{\mathrm{c}}$ at the electrode represent the boundary values which have been used for the discharge currents $i_{L}=320$ and $500 \mathrm{~mA}$, respectively. The modulation of the atom flux at different distances from the electrode roughly corresponds to that of the atom density. However, the ion flux shows a more complicated behavior with positive and negative branches. The backward flux towards the electrode strongly increases during the cathode phase due to the larger density gradient as shown in Fig. 9 which is caused by the enhanced ionization and the larger electric field in the sheath $E_{\mathrm{S}}$.

The ion flux includes the drift flux which depends on the assumed field strength $E_{0}$. The influence of this parameter on the ion density and flux has been analysed by solving the fluid equations also for $E_{0}=-0.4$ and $+0.2 \mathrm{~V} / \mathrm{cm}$. The influence is small in the region between the electrode surface $(r=0.5 \mathrm{~mm})$ and the position where the ion density is maximal $(r \approx 1 \mathrm{~mm})$ because the ion diffusion flux is dominant in this region due to the large density gradient.

The combination of $\mathrm{Ba}$ atom and ion fluxes at the electrode surface allows to determine the net flux of Ba out of the electrode according to

$\Gamma_{\text {net }}(t)=\Gamma_{\mathrm{a}}\left(t, r_{c}\right)-(1-R) \Gamma_{\mathrm{i}}\left(t, r_{c}\right)$

This quantity corresponds to the loss of Ba material which lastly limits the lifetime of the lamp. It is additionally included in the left diagrams of Fig. 10. Due to the increased back flux of ions in the cathode phase the net flux is strongly decreased during this phase.

The temporal evolution of the electron density $n_{e}$ is shown in Fig. 11. The maximal values in the cathode phase are about $2 \cdot 10^{12} \mathrm{~cm}^{-3}$ and $3.5 \cdot 10^{12} \mathrm{~cm}^{-3}$, respecticely, for both cases. Similar 

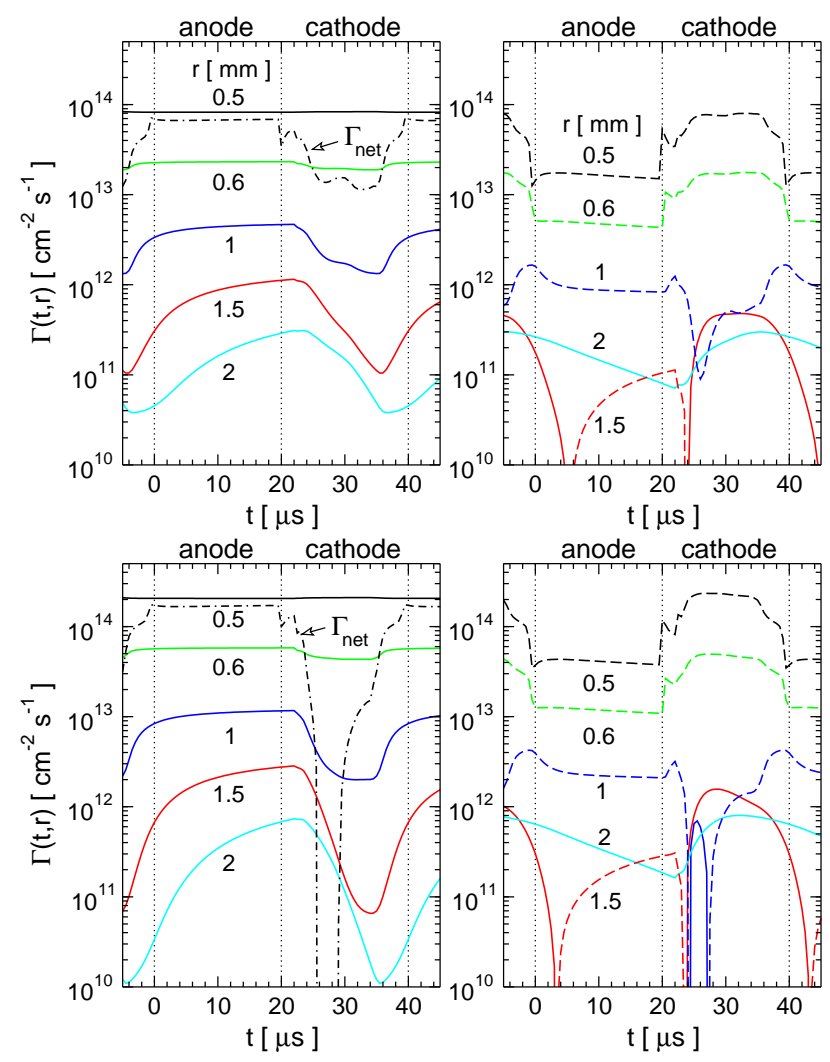

Figure 10. Ba atom (left) and ion (right) fluxes for 320 (top) and $500 \mathrm{~mA}$ (bottom). The dashed curves in the right figures represent negative fluxes. The straight solid lines represent the chosen atom flux $\Gamma_{a}^{c}$ at the spot.
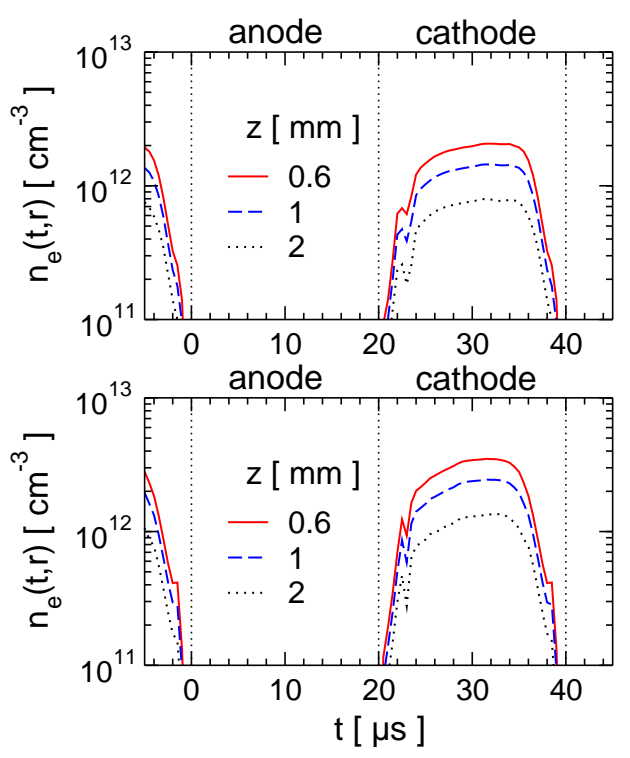

Figure 11. Electron density for 320 (top) and $500 \mathrm{~mA}$ (bottom).

results were obtained in Ref. [4], however with a temporal delay. Due to the quasi-stationary approach of the current model, the electron density is present here in the cathode phase only. However, the main purpose of this investigation is to describe the barium transport in the close vicinity of the spot. In this context, the ionization frequency (11) is the key quantity which peaks with $n_{e}$ and $k_{\mathrm{Ba}}^{\mathrm{io}}$ in the cathode phase.

The impact of the cathode fall voltage is illustrated in Fig. 12. So far a calibration factor of 
$C_{\mathrm{CF}}=1.2$ has been used to relate the measured voltage from the band method to the cathode fall voltage. In Fig. 12 the $\mathrm{Ba}$ atom densities are shown which have been obtained by varying $C_{\mathrm{CF}}$. In both cases the increase of the cathode fall voltage leads to a weaker drop of the Ba density during the cathode phase. This unexpected behavior is caused by the non-monotonous dependence of the $\mathrm{Ba}$ ionization rate coefficient on the cathode fall voltage as shown in Fig. 7. The cathode fall voltage varies between 10 and $12 \mathrm{~V}$ during the main time span $(t=2 \cdots 15 \mu \mathrm{s}$ ) of the cathode phase (cf. Fig. 5) and reaches about $14.5 \mathrm{~V}$ in the peak at about $t=23 \mu \mathrm{s}$. Around this cathode fall voltage the ionization rate coefficient decreases with increasing voltage. At the higher discharge current $i_{L}=500 \mathrm{~mA}$ the results obtained for $C_{\mathrm{CF}}=1.0$ and 1.2 nearly agree because the ionization rate coefficient only weakly changes around the averaged cathode fall voltage of about $U_{\mathrm{CF}}=10.5 \mathrm{~V}$.
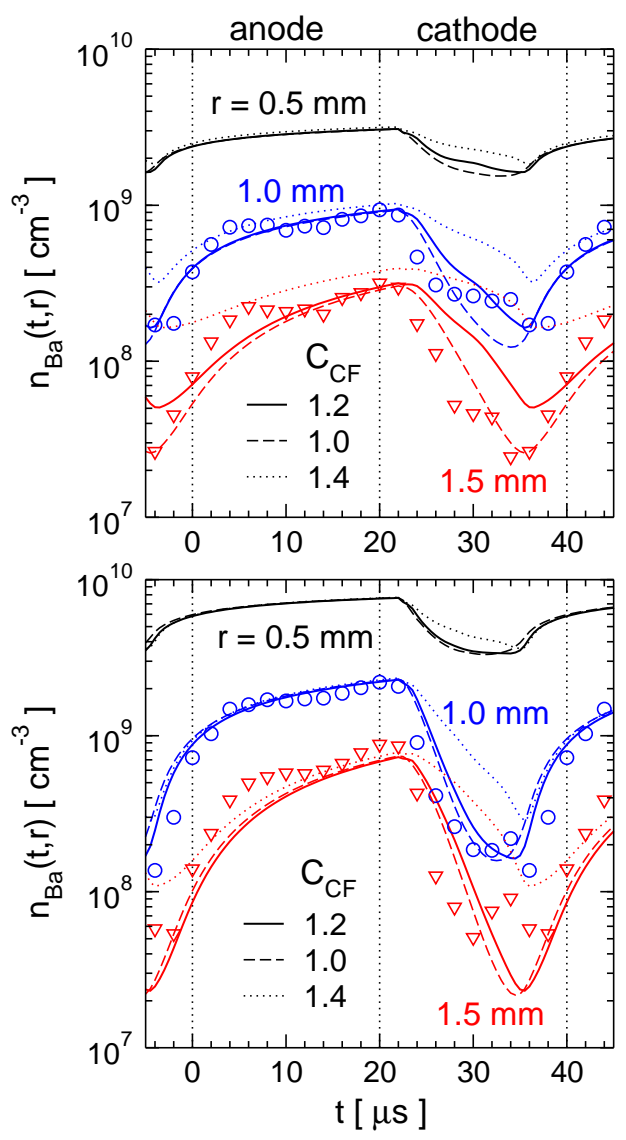

Figure 12. Ba atom density for different values of the calibration factor $C_{\mathrm{CF}}$ for 320 (top) and 500 $\mathrm{mA}$ (bottom).

\section{Conclusion}

The temporal and spatial behaviour of $\mathrm{Ba}$ atom and ion densities originating from the spot evaporation at the coiled electrode of a commercial fluorescence lamp in ac operation at $25 \mathrm{kHz}$ has been studied. The determination of Ba evaporation flux and total Ba loss over the whole period of the ac operation became possible due to the combination of absolute calibrated LIF measurements, estimations of the cathode fall and the coupling with an electron kinetic and a $\mathrm{Ba}$ atom and ion transport model. The distinct impact of the diffusion and of the ionization by plasma electrons in the cathode phase has been revealed. The diffusion is influenced by the gas temperature profile around the spot too. In the frame of the model the non-equilibrium and nonlocal 
behavior of the electron component in the spot region is reflected in the nonlinear dependence of the ionization frequency on the electrode fall voltage. Despite of this, the fall voltage magnitude is of lower importance for the estimation of the Ba evaporation flux. The good agreement of the temporal and spatial course of the densities obtained by the model and the LIF measurements reveals the accuracy of the measurements and confirms the assumptions made in the modeling. Because the study omitted a detailed description of the evaporation itself, it further on enables a comparison with results of electrode and evaporation models as well as their validation together with measurements of the electrode temperature distribution. The latter will be subject of future investigations.

\section{Acknowledgments}

This work was supported by Osram Munich.

\section{References}

[1] Bhattacharya A K 1989 Journal of Applied Physics 65 4595-4602

[2] Hilbig R and Chittka U 1989 Proc. 5th Int. Symp. on the Science and Technology of Light Sources ed Devonshire R, Meads J and Wharmby D O (York, UK) pp 245-246

[3] Hadrath S, Beck M, Garner R, Lieder G and Ehlbeck J 2007 J. Phys. D: Appl. Phys. 40 163-167

[4] Garner R 2008 J. Phys. D: Appl. Phys. 41 144010(20pp)

[5] Porokhova I A, Winter J, Sigeneger F, Loffhagen D and Lange H 2009 Plasma Sources Sci. Technol. 18015013

[6] Ralchenko Y, Kramida A, Reader J and Team N A 2008 NIST Atomic Spectra Database (version 3.1.5)

[7] Hadrath S, Ehlbeck J, Lieder G and Sigeneger F 2005 J. Phys. D: Appl. Phys. 38 3285-3295

[8] Garner R 2008 J. Phys. D: Appl. Phys. 41 144009(13pp)

[9] Nachtrieb R, Khan F and Waymouth J F 2005 J. Phys. D: Appl. Phys. 38 3226-3236

[10] Bartschat K, privat communication

[11] Hayashi, M. 1992, Plasma Material Science Handbook Appendix 3, ed Japan Society for the Promotion of Science (Tokyo: Ohmsha)

[12] Sigeneger F, Winkler R and Robson R E 2003 Contrib. Plasma Phys. 43 178-197

[13] Fursa D, Bray I, Csanak G, Clark R, Abdallah J, Kanik I and Trajmar S 2002 Phys. Rev. A 65032723

[14] Sigeneger F, Sukhinin G and Winkler R 2000 Plasma Chem. Plasma Process. 20 87-110

[15] Lister G and Coe S 1993 Comp. Phys. Commun. 75 160-184

[16] Boeuf J P and Marode E 1982 J. Phys. D: Appl. Phys. 15 2169-2187

[17] Sirghi L, Ohe K and Popa G 1998 J. Phys. D: Appl. Phys. 31 551-560

[18] Namiotka R, Ehrlacher E, Sagle J, Brewer M, Namiotka D, Hickman A, Streater A and Huennekens J 1996 Phys. Rev. A $\mathbf{5 4} 449-461$

[19] Viehland L A and Mason E A 1995 At. Data and Nucl. Data Tables 60 37-95

[20] Hagelaar G, Hoog F and Kroesen G 2000 Phys. Rev. E 62 1452-1454 